\title{
MEDITERRANEAN SPOTTED FEVER IN CHILD AND ADULT PATIENTS: INVESTIGATION FROM AN ENDEMIC REGION IN BULGARIA
}

\author{
Ivan Baltadzhiev', Ani Kevorkyan², Nedialka Popivanova ${ }^{3}$ \\ ${ }^{1}$ Department of Infectious Diseases, Parasitology and Tropical Medicine, Medical University, University Clinic of Infectious Diseases at \\ St. George University Hospital, Plovdiv, Bulgaria \\ ${ }^{2}$ Department of Epidemiology and Medicine of Disastrous Events, Medical University, Plovdiv, Bulgaria \\ ${ }^{3}$ Medical University, Plovdiv, Bulgaria
}

\section{SUMMARY}

Objectives: Mediterranean spotted fever (MSF) is a tick-borne endemic disease caused by Rickettsia conorii conorii and transmitted to humans by the brown dog tick Rhipicephalus sanguineus. It is characterized by fever, maculopapular rash and a tick bite skin lesion "tache noire". The disease affects all age groups and usually has mild to moderate course. It has long been considered a benign infection. However, very severe "malignant" forms with fatal outcome strongly influenced by the patients' age were also observed. We aimed to reveal the features of MSF in children by comparing them with some of the disease patterns in adults.

Methods: Our prospective study data are based on the monitoring of all inpatients of the Clinic of Infectious Diseases in Plovdiv City and environs, the largest MSF endemic region in Bulgaria. The total number of patients admitted and treated for MSF over a study period of 10 years is 549 , the incidence reaching $9.44 / 100,000$. MSF is confirmed by antibody response to a specific antigen measured by indirect immunofluorescence assay (IFA). $\lg \mathrm{g} \geq 128$ and/or $\lg M>64$ were considered to be indicative of acute infection.

Results: Eighty-five (15.48\%) patients were children up to 14 years of age and 464 (84.52\%) were aged 15-85 years. The skin lesion "tache noire" was present in $73.68 \%$ of children, and in $76.93 \%$ of the older age groups; symptoms as anorexia, nausea, vomiting, abdominal pain, and diarrhoea were more frequent in children, till general weakness and malaise was prevalent in adults. Children accounted approximately for $70 \%$ of the disease mild forms. No lethality was registered in this age group. Even though there were isolated cases of severe and malignant forms, children did not undergo the fatal complications seen in adults.

Conclusion: Knowledge of the peculiarities of MSF in children will contribute to children's health protection, timely diagnose and disease prevention.

Key words: Mediterranean spotted fever, Boutonneuse fever, Rickettsia conorii, children

Address for correspondence: I. Baltadzhiev, Department of Infectious Diseases, Parasitology and Tropical Medicine, Faculty of Medicine, Medical University, 15A Vassil Aprilov Blvd., 4002 Plovdiv, Bulgaria. E-mail: ivan_balt@yahoo.com

https://doi.org/10.21101/cejph.a5608

\section{INTRODUCTION}

Mediterranean spotted fever (MSF), also known as Marseilles fever (MF), or Boutonneuse fever (BF) is caused by Rickettsia conorii conorii, strain Malish (1). Rickettsia conorii is a small, gram-negative, rod-shaped bacteria, whose sizes range from $0.3-2.0 \mu \mathrm{m}$. It is strictly intracellular pathogen, belonging to the spotted fever group (SFG) of the genus Rickettsia in the family Rickettsiaceae. The SFG rickettsiae are natural parasites of certain arthropods, e.g. R. conorii is transmitted to humans by the brown dog tick Rhipicephalus sanguineus, assumed to be both vector and reservoir of infection. This is the most common tick species along the Mediterranean coastal regions as well as in several other European countries. Natural hosts of $R$. sanguineus are stray and pet dogs. They play a significant role in the epidemiological chain as intermediaries between ticks and humans.

Pathogenic rickettsiae infect and replicate in vascular endothelial cells lining the capillaries, arterioles, and venules. Their tropism towards microvascular endothelium leads to endothelial cell activation, disseminated inflammation and altered vascular functions, consisting of compromised vascular integrity, increased permeability and loss of barrier role. The endothelial dysfunction during rickettsial infection, termed rickettsial vasculitis manifests as variety of organ systems impairment and complications.

In Bulgaria, I. Vapzarov described the first cases occurring as a small outbreak in the Plovdiv region (2). The emerging disease was sporadic and largely occurring in mild or moderate form. Over a period of approximately 20 years (1972-1992) new cases of MSF had not been registered. Early in 1993, MSF re-emerged with new distinctive features as spread to new geographical areas; predominance in urban residents; and presence of more severe clinical forms resulting in complications and even deaths. The disease maintained its classic clinical picture: an eschar at the site of tick bite (termed "tache niore"), an abrupt onset with fever, followed by flu-like symptoms preceding the development of diffuse maculopapular rash spreading over the trunk and extremi- 
ties, involving the palms and feet. All age groups were affected, from infancy to late adulthood with significant differences in the severity and outcomes among younger and older patients.

The purpose of the study was to outline the features of MSF in children by comparing them with some clinical patterns of the disease in adults - basic symptoms and signs, severity, organ systems involvement and outcome.

\section{MATERIALS AND METHODS}

Our prospective study data are based on the monitoring of all inpatients of the Clinic of Infectious Diseases at the St. George University Hospital - the only medical facility serving and treating patients with MSF in Plovdiv City and suburbs, the largest endemic MSF region in Bulgaria with approximately 700,000 inhabitants. The total number of patients admitted and treated for MSF over a study period of 10 years is 549, the incidence reaching $9.44 \pm 1.82$ (SEM) per 100,000, complying with the regular census data for Plovdiv region. Eighty-five (15.48\%) of the patients were children under the age of 15 years (range from 1 month to 14 years of age) and $464(84.52 \%)$ were aged $15-85$ years. Although childhood formally refers to the period between infancy and 18 years of age, we considered the peculiarities of the disease in children at the age of 14 . The reason was that in the 15-19 age group the clinical picture of MSF was closer to that of the older (20-34), than to the group comprising infants, toddlers, preschool children, and adolescents under the age of 15 years. In order to be maximally objective, MSF severity was determined in age groups with an equal year number (14-year interval): 0-14, 20-34, 40-54, and 60-74. Most epidemiological criteria and criteria determining the severity of MSF have been detailed in our previous studies $(3,4)$. The severity criteria are only briefly outlined herein:

Mild form: mild or absent toxic-infectious syndrome (TIS)*; fever about $38-38.5^{\circ} \mathrm{C}$, no chills or intensive headaches; moderate myalgia and arthralgia; scarce or less abundant maculopapular rash; no significant deviations in the laboratory parameters (mild variations in peripheral white blood count, platelet count about $120-140 \times 10^{9} / 1$ or more; might happen a negligible increase in aminotransferase /ALT and AST/ activity).

Moderate form: well pronounced toxic-infectious syndrome (TIS), fever about $38.5-39^{\circ} \mathrm{C}$, chills, no or tolerable headache; moderate or abundant maculopapular rash (mostly non-petechial); $\mathrm{X}$-rays indicative of bronchitis or peribronchial changes; minor hepatomegaly; typical laboratory findings: moderate leukocytosis/ leukopenia with shift to the left; ALT and AST 2 to 3-fold increase in the activity; platelet count of $\geq 100 \times 10^{9} / 1$; serum sodium at the lower reference limit (136-135 mmol/l); serum albumin at the lower reference limit $-35 \mathrm{~g} / \mathrm{l}$; blood urea nitrogen (BUN) and creatinine: within the reference range or mildly elevated.

Severe form: severe TIS, temperature over $39^{\circ} \mathrm{C}$, chills, severe headache, repeated vomiting; abundant rash with haemorrhagic characteristics over the lower extremities or over the trunk; clinically and laboratory manifested damage to a single body organ (lung, liver, kidney, myocardium, etc.); platelet count of $>50$ $<120 \times 10^{9} / 1,\left(\leq 100 \times 10^{9} / 1\right.$ in most cases $)$; serum sodium $<136$ $\mathrm{mmol} / \mathrm{l} ; \mathrm{Ca}<2.12 \mathrm{mmol} / \mathrm{l}$; ALT/AST elevation $\geq 4-5$ times; BUN and creatinine - above reference levels.

Malignant form: very severe TIS, temperature $\geq 40^{\circ} \mathrm{C}$, chills, severe headache, nausea, vomiting, typhoid mental state, stupor or coma; abundant haemorrhagic rash; clinically apparent lesions of more than one organ (pneumonitis, jaundice, renal failure, gastrointestinal haemorrhage, myocarditis, CNS damage); platelet count $\leq 50$ or $<100 \times 10^{9} / 1$; serum sodium $\leq 130 \mathrm{mmol} / \mathrm{l}$; ALT/AST $>5$ fold elevation, hypocalcaemia, hypoalbuminemia, hypoxemia.

Patients were enrolled in the study based on the following inclusion criteria: development of febrile illness with flu-like symptoms 3-4 days prior to onset of the typical maculopapular rash; the pathognomonic eschar (tache noire) or other suspected port of entry - conjunctiva, skin lesions, etc.; at least 4-fold rise of the $R$. conorii antibody titer in repeated serum samples or suitably increased IgG and/or IgM titer; and no anti-rickettsial antibiotic treatment before admission. Exclusion criteria: patients with coexisting acute or exacerbated chronic medical conditions were excluded from the study.

The disease was diagnosed based on epidemiological and clinical criteria and aetiologically confirmed by positive antibody response to the $R$. conorii specific antigen, measured by the indirect immunofluorescence assay (IFA) performed at the Reference Rickettsioses Laboratory at the Military Medical Academy - Sofia (Rickettsia conori-Spot IF, BioMerieux, Marcy L'Etoile, France). IgG $\geq 128$ and/or IgM $>64$ were considered to be indicative of acute infection. Treatment was based on macrolides in children under eight and doxycycline in grown up children and adults.

\section{Statistics}

Data were analyzed using the SPSS statistical software 15 (SPSS Inc., Chicago, IL). Fisher's exact test determining the two-tailed p-values and the $95 \%$ confidence intervals (CIs) was used to differentiate between the age groups for clinical signs and symptoms, and the severity of MSF. Two-tailed $\mathrm{p}<0.05$ was considered statistically significant.

\section{RESULTS}

The age distribution of MSF patients is presented in Figure 1. Children at the age of 14 accounted for approximately $15 \%$ of the total MSF patients over the studied 10-year period. Interestingly, among all children the percentage of toddlers up to 4 years of age was the highest, followed by young children aged 5-9 years, and the percentage of adolescents 10-14 years old was the smallest (Fig. 1).

The primary skin lesion "tache noire de Pieri" was present nearly as frequently in children $-73.68 \%$, as in the remaining MSF age group $-76.93 \%(\mathrm{p}>0.05)$. The tick-bite lesion in our patients did not differ significantly from the described classic one: a small,

\footnotetext{
*Toxic-infectious syndrome includes all or some of the following symptoms: fever, chills, headache, arthralgia, myalgia, malaise (adynamia), dizziness, anorexia, nausea, vomiting, abdominal pain, diarrhoea, etc.
} 


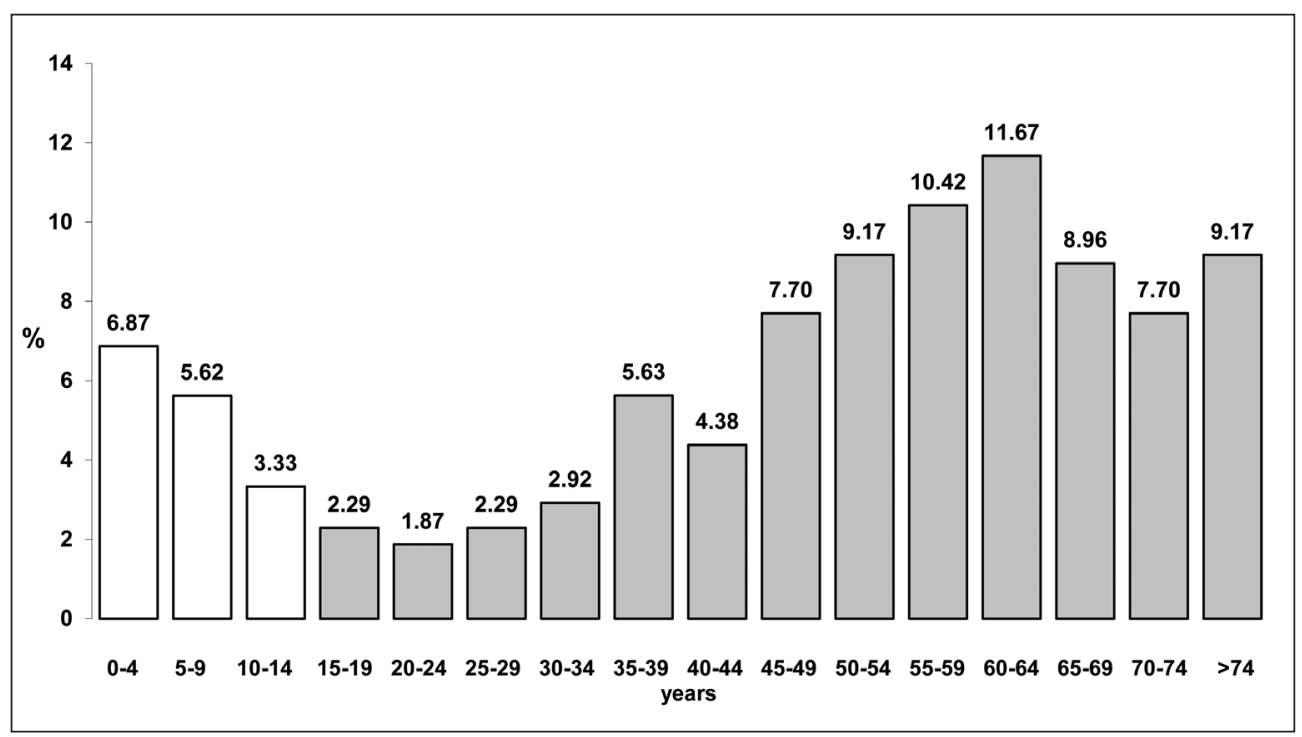

Fig. 1. Age groups of patients with Mediterranean spotted fever.

irregularly rounded or slightly elongated red skin infiltrate with a medial black crust, not itchy, painless, and most often without lymphangitis or regional lymphadenitis in adult patients (except in cases with secondary infection present). In children, however, tache noire was often accompanied by regionally enlarged, elastic and moderately painful lymph nodes (39.2\%). Localization of the skin eschar was variable and occasionally unusual. Statistically significant difference in children compared to adults was observed regarding the lesion location: in youngsters, it was most often found on the head, neck, genitals and in the auricle. For example, nearly half of the tick bites in children affected the head-neck-ear region $(41.16 \%) ; 18.80 \%$ fall on the upper parts of the body (arm, shoulder, chest, back); about a quarter (27.03\%) of the eschars were positioned in the navel area, in the lumbar, buttocks, and genitalia region; the lower parts of the body (thighs, lower leg, ankle) accounted for the remaining $12.92 \%$. In adults, the eschar was more common in the axilla, the upper limbs, inguinal fold, and lower limbs (Table 1).

Table 1. Comparative presentation of tache noire distribution in adults and children with Mediterranean spotted fever ( $N=549)$

\begin{tabular}{|c|c|c|c|c|c|}
\hline \multirow[t]{2}{*}{ Tache noire localization } & $\begin{array}{l}\text { Adults } \\
n=464\end{array}$ & $\begin{array}{c}\text { Children } \\
n=85\end{array}$ & \multirow[t]{2}{*}{$\mathrm{p}$-value } & \multirow[t]{2}{*}{ Odds ratio } & \multirow[t]{2}{*}{$95 \% \mathrm{Cl}$} \\
\hline & $n(\%)$ & $n(\%)$ & & & \\
\hline Head $^{*}$ & $16(3.44)$ & $18(21.17)$ & $<0.001$ & 0.133 & $0.065-0.273$ \\
\hline Neck* & $17(3.66)$ & $13(15.29)$ & $<0.001$ & 0.211 & $0.098-0.452$ \\
\hline Auricle & $8(1.72)$ & $4(4.70)$ & 0.09 & 0.355 & $0.105-1.208$ \\
\hline Breast & $39(8.40)$ & $6(7.05)$ & 0.83 & 1.208 & $0.495-2.950$ \\
\hline Back & $48(10.34)$ & $6(7.05)$ & 0.43 & 1.519 & $0.629-3.671$ \\
\hline Lumbar region & $19(4.09)$ & $4(4.70)$ & 0.76 & 0.865 & $0.287-2.608$ \\
\hline Belly & $35(7.54)$ & $6(7.05)$ & 1.00 & 1.074 & $0.437-2.639$ \\
\hline Gluteal region & $45(9.69)$ & $6(7.05)$ & 0.54 & 1.414 & $0.583-3.427$ \\
\hline Axilla and upper limb* & $57(12.28)$ & $4(4.70)$ & 0.03 & 2.836 & $1.001-8.037$ \\
\hline Inguinal fold & $28(6.03)$ & - & - & - & - \\
\hline Thigh & $39(8.40)$ & $5(5.88)$ & 0.52 & 1.468 & $0.561-3.840$ \\
\hline Patella & $4(0.86)$ & - & - & - & - \\
\hline Behind-knee well & $10(2.15)$ & - & - & - & - \\
\hline Lower thigh & $47(10.12)$ & $3(3.52)$ & 0.06 & 3.081 & $0.936-10.140$ \\
\hline Ankle & $14(3.01)$ & $3(3.52)$ & 0.73 & 0.850 & $0.239-3.026$ \\
\hline Foot & $13(2.80)$ & - & - & - & - \\
\hline Mons pubis & $10(2.15)$ & - & - & - & - \\
\hline Scrotum/prepuce*/vulva & $13(2.80)$ & $7(8.23)$ & 0.02 & 0.321 & $0.124-0.831$ \\
\hline
\end{tabular}

${ }^{*}$ Statistical significant difference; $\mathrm{Cl}$ - confidence interval; two-tailed Fisher's exact test 
The incubation period from the tick bite to the onset of first symptoms in child and adult patients fluctuated within comparable time range and was most often 5 (40.74\%) to 7 (34.15\%) days.

The main symptoms of MSF occurred 3-5 days before the appearance of typical maculopapular rash. They are comparatively presented between children and adults in Table 2. The symptoms of MSF were similar in both age groups however some of them were more common in adult patients compared to children and vice versa. These symptoms included malaise (adynamia) which was far more frequent in adults, whereas gastrointestinal symptoms as appetite loss, nausea, vomiting, abdominal pain, and diarrhoea were significantly more common in children, and the latter applies to sore throat too. Enlarged liver and spleen were seen in $63.15 \%$ of the children versus $13.84 \%$ of the older age group. Isolated liver enlargement was observed in $23.68 \%$ of children versus $46.44 \%$ of adults (Table 2 ).

The rash is one of the most typical features of MSF. In our observations it had a classic pattern: maculopapular, scarce or abundant, unequal in size and prominence, at times progressing to petechial or haemorrhagic, the latter seen typically in the most severe forms of the disease. It rarely involved the face, diffusely spread to the body and limbs without sparing the palms and feet. There were no particular differences in the morphology and location of the rash observed in children and adults suffering from MSF, but in children no haemorrhagic pattern was seen, and rarely a petechial component to the rash units was noticed.

As for the severity of the disease, the mild forms were 226 $(41.16 \%)$, moderate $180(32.79 \%)$, and severe $88(16.03 \%)$. Malignant forms were $55(10.02 \%)$ and $19(34.5 \%)$ of them were lethal. In Figure 2, children were compared separately to patients of the other three age groups in terms of the MSF severity. The percentage of mild MSF cases in children until the age of 15 years significantly exceeded that recorded ones in the 40-54 and 60-74 age groups. For example, in the group of patients with mild MSF the proportion of children up to 15 years was $69.4 \%$, while the proportion of adults over 40 and over 60 was $38.7 \%$ and $27.8 \%$, respectively. In both cases was $\mathrm{p}<0.0001$ (Fig. 2). Similar tendency, however, in the opposite direction, was observed regarding the percentage of moderate and severe MSF forms - they were predominant in the older age groups. Regarding the malignant forms, the difference was statistically significant between the youngest and the oldest age group: malignant MSF was present in a very small proportion of children and was nearly three times more common in elderly patients aged 60-74 years. No statistically significant difference was found between the relative share of children and young people aged 20-34 in either of the abovementioned MSF forms of severity.

Regarding the studied clinical and laboratory parameters, they corresponded to the criteria for MSF severity and reflected the prevailing share of mild and moderate disease forms in children, with a small percentage of severe and malignant forms among them. In children we recorded 4 (4.71\%) severe MSF cases and 4 (4.71\%) malignant cases, however, no fatal outcomes were seen. Interstitial pneumonia was detected in four children with severe MSF, while the children with malignant form of the disease presented with one of them in the course of MSF manifested glucose-6-phosphate dehydrogenase (G6PD) deficiency with severe haemolysis and multiorgan involvement; two children had severe liver involvement with hyperbilirubinemia and increased aminotransferase activity, along with thrombocytopenia, hypoproteinemia, and hyponatremia; one child developed altered consciousness, stiff neck and brain oedema signs. In the cases with liver involvement the markers of viral hepatitis A, B and C were negative. In all that briefly noted cases, the MSF diagnosis was confirmed by an increasing antibody titer (IFA) to $R$. conorii. Despite the severe MSF course all children were discharged from the hospital healthy without any consequences.

Table 2. Mediterranean spotted fever signs and symptoms in adults and children $(N=549)$

\begin{tabular}{|c|c|c|c|c|c|}
\hline \multirow[t]{2}{*}{ Signs and symptoms } & $\begin{array}{l}\text { Adults } \\
n=464\end{array}$ & $\begin{array}{c}\text { Children } \\
n=85\end{array}$ & \multirow[t]{2}{*}{$p$-value } & \multirow[t]{2}{*}{ Odds ratio } & \multirow[t]{2}{*}{$95 \% \mathrm{Cl}$} \\
\hline & $\mathrm{n}(\%)$ & $n(\%)$ & & & \\
\hline Fever & $460(99.14)$ & $85(100)$ & 1.00 & 0.598 & $0.032-11.220$ \\
\hline Myalgia, arthralgia & $344(74.13)$ & $58(68.23)$ & 0.28 & 1.334 & $0.808-2.204$ \\
\hline Malaise ${ }^{\star}$ (adynamia) & $317(68.31)$ & $46(54.11)$ & 0.01 & 1.828 & $1.143-2.924$ \\
\hline Chills & $313(67.45)$ & $51(60.00)$ & 0.21 & 1.382 & $0.859-2.223$ \\
\hline Anorexia* & $266(57.32)$ & $60(70.58)$ & 0.02 & 0.560 & $0.339-0.924$ \\
\hline Headache & $156(33.62)$ & $29(34.11)$ & 1.00 & 0.980 & $0.600-1.594$ \\
\hline Vomiting* & $82(17.67)$ & $45(52.94)$ & $<0.001$ & 0.191 & $0.117-0.311$ \\
\hline Nausea* & $52(11.20)$ & $43(50.58)$ & $<0.001$ & 0.123 & $0.074-0.206$ \\
\hline Abdominal pain* & $31(6.68)$ & $20(23.52)$ & $<0.001$ & 0.233 & $0.125-0.432$ \\
\hline Backache & $18(3.87)$ & $2(2.35)$ & 0.75 & 1.675 & $0.381-7.357$ \\
\hline Dizziness & $19(4.09)$ & $2(2.35)$ & 0.75 & 1.772 & $0.405-7.754$ \\
\hline Rigid lower limbs & $14(3.01)$ & $4(4.70)$ & 0.50 & 0.630 & $0.202-1.963$ \\
\hline Diarrhea* & $8(1.72)$ & $5(5.88)$ & 0.03 & 0.281 & $0.089-0.880$ \\
\hline Sore throat* & $2(0.43)$ & $7(8.23)$ & $<0.001$ & 0.482 & $0.098-0.237$ \\
\hline
\end{tabular}

*Statistical significant difference; $\mathrm{Cl}$ - confidence interval; two-tailed Fisher's exact test 


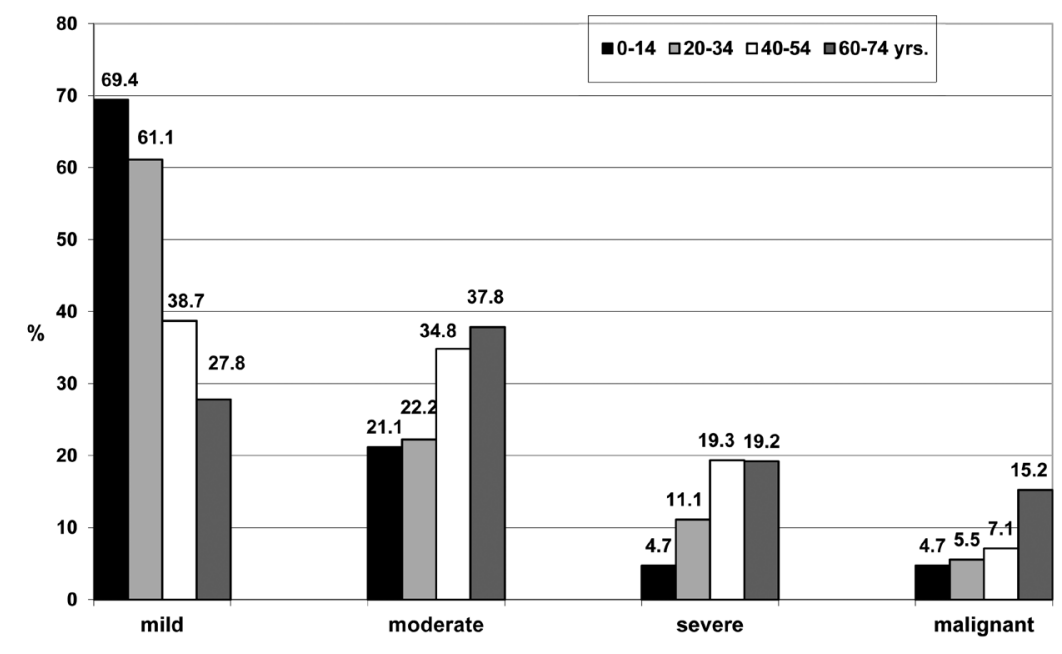

Fig. 2. Forms according to disease severity among child and adult Mediterranean spotted fever patients.

\section{DISCUSSION}

In the last two decades, MSF spread intensively not only in the endemic Mediterranean regions but also in many other European countries, affecting both adult and paediatric populations. In some particularly intensely affected regions, children $<15$ years old accounted for about one-fifth of MSF sufferers annually (5). Approximately in the same proportions were the children among our cohort of patients with MSF. Many authors have dealt with paediatric outcomes in the course of the disease, however, in most cases different childhood age ranges have been studied (6-8). Similar to our study, some authors have preferred to investigate the course of MSF in children under the age of 15 $(5,9,10)$. Closest to our age range are the studies of $\mathrm{C}$. Colomba et al. involving children under 15 years of age as well as other age groups ranging from 15 to 70 years $(5,10)$. These authors have found out that children suffered from milder forms of MSF compared to the adult population - a finding supported by data from our current research, as well as by our previous studies (11). Overall, the acquiring knowledge established in our investigation is that MSF is less common in children under the age of 15 years than in adults over the age of 40 . The reason for this observation is logical: infants, toddlers and preschoolers have a less chance to come in contact with dog ticks, domestic and wild animals; moreover, children are not exposed to the nature and animals as professional hazards.

The inoculation eschar was as common in children as in older age groups. It was more frequently localized in the child's head and neck. The external ear and the genital area in children were predilection sites for tick bites compared to the older age groups. It is likely that the lesser body height allowed easier access of the vectors to the upper trunk and the head. Unlike adults, regional lymphadenitis was more typical for children and the same holds true for the simultaneous enlargement of liver and spleen.

Gastrointestinal symptoms as anorexia, nausea, vomiting, abdominal pain, and diarrhoea were more frequent in children than in adults. Some authors suggest that gastrointestinal symptoms are prominent manifestations in patients with severe/fatal MSF and might be related to central nervous system involvement in case that elevated intracranial pressure was present (12). Regarding children, our data does not support this suggestion. In our opinion, these events are primarily due to the physiological and anatomical features of the digestive system in children, to the special reactivity of their gastrointestinal system, and to their overall emotional instability - a clinical phenomenon seen in other diseases in childhood as well. In the adult patients, abdominal pain was rare and we did not observe diarrhoea, even in the most severe cases. General malaise, however, was more pronounced in older MSF patients.

Children suffered mainly from mild MSF. The analysis of the MSF forms of severity found out that children accounted approximately for $70 \%$ of the mild forms of the disease. No lethalities were registered in this age group. The data of Figure 2 indicate clearly that the severity of the disease increases with advancing age. There are single exceptions which are insufficient to alter the general trends of MSF more severe clinical course with age advancing.

Organ systems involvement exists in varying degree in all forms of MSF due to the typical systemic vascular injury. However, while this involvement might often occur subclinically in mild and moderate forms, seriously manifested disorders occur predominantly in the severe and malignant forms. Despite the milder course of MSF in children, there were isolated cases of severe and malignant forms which seriously affected the whole organism. Four children with interstitial pneumonia experienced severe forms, but ended up with recovery. A malignant form of MSF was observed in a child with G-6-P-DH deficiency, and other two children suffered MSF with severe liver damage. Nervous system was not spared by the rickettsial invasion, both in adults and children (13). There are similar reports in the world literature as well $(14,15)$.

It should be noted that children did not undergo the fatal complications seen in the adults. In previous publications we have described MSF adult patients with various organ systems damages and severe complications as acute respiratory distress syndrome, gastrointestinal bleeding, haemorrhagic-necrotic pancreatitis, 
myocarditis, and acute renal failure $(16,17)$. Similar complications were especially seen in the elderly and not observed in the present child contingent.

From our cohort of 549 patients 19 (3.46\%) ended with a lethal outcome, but none of them was a child. Other authors pointed out as well that the prognosis in paediatric MSF was good (18).

\section{CONCLUSION}

In terms of its clinical manifestations, MSF in children presents itself as a benign disease commonly ongoing without complications. Isolated exceptions do not deny this conclusion. Conversely, in adults aged over 40 , the disease occurs in a more aggressive form and with more complications taking serious, insidious and less predictable course. Knowledge of the specifics of MSF in children will contribute to the timely diagnosis, disease prevention and impact on health in the environment.

\section{Conflicts of Interests}

None declared

\section{REFERENCES}

1. Fournier PE, Raoult D. Intraspecies diversity of Rickettsia conorii. J Infect Dis. 2009;199(7):1097-8.

2. Vapzarov I. About a small epidemic of Fievre boutonneuse in Plovdiv region, Bulgaria. Zdravno delo. 1948;1:21-9. (In Bulgarian.)

3. Baltadzhiev IG, Popivanova NI, Stoilova YM, Kevorkian AK. Mediterranean spotted fever - classification by disease course and criteria for determining the disease severity. Folia Med (Plovdiv). 2012;54(4):53-61.

4. Baltadzhiev IG, Popivanova NI. Some epidemiological features of the Mediterranean spotted fever re-emerging in Bulgaria. Folia Med (Plovdiv). 2012;54(1):36-43

5. Colomba C, Saporito L, Siracusa L, Giammanco G, Bonura S, Titone L. Mediterranean spotted fever in paediatric and adult patients: two clinical aspects of the same disease. Infez Med. 2011;19(4):248-53. (In Italian.)
6. Vitaliti G, Falsaperla R, Lubrano R, Rapisarda V, Cocuzza S, Nunnari G, e al. Incidence of Mediterranean spotted fever in Sicilian children: a clinical-epidemiological observational retrospective study from 1987 to 2010. Int J Infect Dis. 2015;31:35-40.

7. Nafi O, Tarawnah Y, Tarawnah A. Epidemiological evaluation of Mediterranean spotted fever in children of the Karak province in south Jordan. J Infect Dev Ctries. 2017;11(3):242-6.

8. Pishmisheva M, Stoycheva M, Vatev N, Semerdjieva M. Mediterranean spotted fever in children in the Pazardjik region, South Bulgaria. Pediatr Infect Dis J. 2014;33(5): 542-4.

9. Mouffok N, Parola P, Abdennour D, Aouati A, Razik F, Benabdellah A, et al. Mediterranean spotted fever in Algerian children. Clin Microbiol Infect. 2009;15 Suppl 2:290-1.

10. Colomba C, Saporito L, Polara VF, Rubino R, Titone L. Mediterranean spotted fever: clinical and laboratory characteristics of 415 Sicilian children. BMC Infect Dis. 2006;6:60. doi: 10.1186/1471-2334-6-60.

11. Baltadzhiev I, Popivanova N, Dimitrova K, Tsoneva A, Kalinova Z. Mediterranean spotted fever peculiarities in the childhood. In: Tick-born infectious diseases. Plovdiv: Medical University; 2006. p. 139-45. (In Bulgarian.)

12. Sousa R, França A, Dória Nòbrega S, Belo A, Amaro M, Abreu T, et al. Host- and microbe-related risk factors for and pathophysiology of fatal Rickettsia conorii infection in Portuguese patients. J Infect Dis. 2008;198(4):576-85.

13. Baltadzhiev I, Boev I, Dineva A, Gocheva A, Kazakova T, Tsoneva A. Meningitis and meningoencephalitis caused by Rickettsia conorii. Med Rev. 2013;49(2):73-7. (In Bulgarian.)

14. Bougteba A, Basir A, Charradi N. Meningoencephalitis caused by Rickettsia conorii in a young infant. Rev Neurol (Paris). 2011;167(2):173-6. (In French.)

15. Tikare NV, Shahapur PR, Bidari LH, Mantur BG. Rickettsial meningoencephalitis in a child--a case report. J Trop Pediatr. 2010;56(3):198-200.

16. Baltadzhiev I, Popivanova N, Zaprianov Z. Malignant forms of Mediterranean spotted fever: risk factors for fatal outcomes. Braz J Infect Dis. 2016;20(5):511-2.

17. Baltadzhiev I, Popivanova N, Zaprianov Z. Hemorrhagic-necrotizing pancreatitis - an unusual complication of Mediterranean spotted fever IOSR J Dent Med Sci. 2017;16(3 Ver I):109-12.

18. Garnier JM, Jurquet AL, Retornaz K, Fournier PE, Minodier P. Pediatric Mediterranean spotted fever. Arch Pediatr. 2009;16 Suppl 2:S93-5. (In French.)

Received November 20, 2018 Accepted in revised form April 14, 2020 